I believe there is a danger that this operation, which is in so many respects satisfactory, may be brought into disrepute, and perhaps even prematurely abandoned, because of the number of vascular necrotic complications, many of them fatal, now being reported. Professor Johnston collected 10. It is my belief that this complication occurs following a traumatic operative procedure and may be preventable. I believe that it will not occur if the dissection along the lesser curve is performed with sufficient care and skill, if diathermy is not used, if the continuity of the greater curve and lesser omentum is not breached, and if the opening into the lesser sac, created during the procedure, is repaired. It has been my practice during the past three years to reperitonealise the lesser curve using 4-5 interrupted Dexon OOO sutures, the ends of which are left long, but in addition I also suture together the anterior and posterior edges of the separated lesser omentum with similar material, again leaving these edges long. These two sets of sutures are serially tied to reattach the lesser omentum to the lesser curve. The postoperative appearance is almost as if surgery had not taken place.

I further suggest that this operation could take the place that partial gastrectomy held in the 1950 s as a training operation for young surgeons, but it is not an operation to be done haphazardly. I think that all future cases of lesser-curve necrosis should be reported, including details as to the exact nature of the procedure and any operative complications. Only then can this rare but frightening complication be seen in true perspective.

SEAN J HefFernaN

Mater Misericordiae Hospital,

Mater
Dublin

\section{Management of uraemic pericarditis}

SIR,-The article by Dr P Kramer and others (6 December 1975, p 564) suggests that cardiac tamponade is almost to be expected when haemodialysis rather than peritoneal dialysis is used for uraemic patients who have pericarditis. It is, however, unfortunate that the authors do not state whether regional or systemic heparinisation was used during haemodialysis. If systemic heparinisation was used their results are perhaps to be expected.

In our unit we use regional heparinisation during dialysis routinely when pericarditis develops, and our experience contrasts with that of Dr Kramer and his colleagues. When regional heparinisation is performed the clotting time of the patient is measured cvery 15 minutes and the aim is to maintain this as near normal as possible by appropriate adjustment of the rate of heparin and protamine infusion. Although this method of heparinisation is not fool-proof, it is usually possible to maintain the patient's clotting time very close tonormal. In patients presenting with untreated end-stage renal failure and pericarditis the lesion has almost universally resolved when twice-weekly haemodialysis with regional heparinisation has been started. We have also experience with the use of regional heparinisation in patients who have been established on intermittent haemodialysis with systemic heparinisation and have subsequently developed pericarditis. Out of seven such patients who had pericarditis with an effusion (detected by echocardiography) two developed cardiac tamponade and required pericardiectomy (both are still alive), but in the other five the effusion resolved spontaneously. In all these cases haemodialysis was performed using regional heparinisation from the time that pericarditis was first detected until the pericardial effusion was shown by echocardiography to have resolved.

Since the risk of cardiac tamponade with haemodialysis is not related to dialysis as such but to heparinisation on dialysis we feel that the method of heparinisation should be stated before concluding that pericarditis will not resolve with haemodialysis. In our experience pericarditis certainly can resolve with continuing haemodialysis using regional heparinisation.

R WINNEY

Medical Renal Unit, Royal Infirmary,

Edinburgh

\section{The problem of rosacea}

SIR,-I was interested to see Dr R Marks's suggestion (10 January, p 94) that dermal solar elastosis may be the initiating factor in rosacea. Surely if this is the case white inhabitants of high-sunshine climates, in whom solar elastosis is so common, would have a very high incidence of rosacea. Personal observations ${ }^{1}$ did not show this, nor am I aware of any studies which have.

Dr Marks proposes that lymphoedema may cause irritating substances to leak into the dermis and give rise to the inflammatory changes of rosacea. If this is the case facial lymphoedema secondary to hypoplastic lymphatics would result in rosacea-like skin lesions, whereas recurrent erysipelas is the common complication. ${ }^{2}$

The recent correlation between rosacea and migraine by Drs S G Tan and W J Cunliffe (3 January, p 21) would strengthen the hypothesis that rosacea is probably an excess vasodilatation in response to many stimuli mediated by unidentified chemicals, and lymphoedema and solar elastosis are secondary phenomena.

Dublin

Charles Dupont

' Dupont, C, British fournal of Clinicai Practice, 1968, ${ }^{2}$ Borrie, P, and Taylor, G W, British fournal of Dermatology, 1962, 74, 403 .

\section{Steroids and common skin diseases}

SIR,-We should like to reply to Dr K Haeger's comments (17 January, p 155) on our article (1 November, p 264). The preliminary results we obtained with Miol lotion and cream in 120 patients were published as a "short report," the maximum length of which is 600 words. We had no space therefore to describe the previous treatments received by these patients and we could do no more than summarise the management and progress of each patient.

Briefly, all the patients had been treated by their general practitioners and then as hospital outpatients for at least a month, using corticosteroids topically; in addition antibiotics had been included in the skin medicaments by the GP in almost three-quarters of the cases. All the patients had failed to show an adequate response or had relapsed early in the course of their treatment. The extent of their skin disease and their refractoriness to treatment fully justified their admission to hospital.
They were then treated as inpatients with carefully supervised corticosteroid applications and, in the case of varicose ulcers, with appropriate compression bandaging and limb elevation. As in the $20 \%$ or so of patients who failed to respond to $\mathrm{Dr}$ Haeger's equally assiduous treatment, the patients included in our report continued to defy our therapeutic attempts after we had persisted in each case for an adequate length of time. We therefore stopped the existing treatment except, as stated in our article, for the "physical" treatment of the leg ulcers. The gratifying response to the Miol preparations in 96 of these very difficult cases stimulated us to report our results.

Although Dr Haeger has not seen ill effects in patients with varicose eczema treated with potent corticosteroids-presumably under his personal supervision-our experience has been less reassuring. Many of the patients we see have been treated previously at home for prolonged periods with corticosteroids; in such cases adverse effects are not uncommon. We are also very concerned to find that valuable systemic antibiotics are often used in skin medicaments without bacteriological monitoring. As a result of this we have encountered alarming instances when patients' chronic skin lesions have, at the time of admission to hospital, yielded heavy cultures of organisms such as Staphylococcus aureus resistant to fusidic acid and Pseudomonas aeruginosa resistant to gentamicin. We feel that only our special interest in the problem of bacterial dispersal from the skin has enabled us to prevent dangerous dissemination of such organisms throughout the hospital environment.

S SELWYN

PeTER Copeman

Departments of Bacteriology

and Dermatology,

estminster Hospital and Medical

London SW

\section{Value of clinical pharmacology}

SIR,-In your leading article on antirheumatic drugs (10 January, p 59) you make short work of specialists in pharmacotherapeutics who are "engrossed in drug kinetics" and "drug interactions of dubious clinical relevance," and correctly so if this can be seen to be the sole preoccupation of all such specialists.

However, in defence of drug kinetics, an understanding of the ways in which drugs are absorbed, distributed, metabolised, and eliminated from the body, of the time course of their effects, and of the alterations that may be produced by disease may influence radically the design of treatment regimens. To take an obvious example, the oral dose of a drug that is eliminated predominantly by the kidney may need to be modified greatly if severe toxicity is to be avoided in a patient with renal failure. The production of a dosage schedule that is both safe and effective may demand the application of kinetic principles.

Drug interactions have become a fashionable topic for study and many of those reported have been of "dubious clinical relevance." Nevertheless, there are some that are already known to be extremely important in practice (for example, interactions with anticoagulants or with potent drugs acting on the cardiovascular system) and much current researcheven the apparently irrelevant-may be justified if it throws light on the mechanisms that determine the way one drug alters the activity of another. 